\title{
THE OSCILLATION OF AN OPERATOR
}

\author{
BY \\ ROBERT WHITLEY
}

\begin{abstract}
Foiaş and Singer introduced the oscillation of a bounded linear operator mapping $C(S)$ into a Banach space. Using this concept we define a generalization of the Fredholm operators $T$ with $\mathscr{K}(T)<\infty$ and a corresponding perturbation class which contains the weakly compact operators. We show that a bounded linear operator on $c$ is a conservative summability matrix which sums every bounded sequence if and only if it has zero oscillation at infinity.
\end{abstract}

The oscillation of a bounded linear operator mapping $C[0,1]$ into a Banach space $X$ is defined in [6] and is used there to discuss two classes of operators, almost diffuse operators and countably almost diffuse operators, each class containing the weakly compact operators. These operators have recently been useful in approximation theory $[2, \S 3]$.

In the following $S$ will always denote a compact Hausdorff space, $X$ a Banach space, and we will consider the bounded linear operators mapping $C(S)$ into $X$. Such operators have interesting properties which can be defined in terms of their oscillation: a generalized notion of a Fredholm operator $T$ with index $\mathscr{K}(T)<\infty$, a corresponding perturbation class and a basic type of conservative summability method.

We restate the definitions of Foiaș and Singer [6]. Let $T: C(S) \rightarrow X$ be a bounded linear operator, the oscillation of $T$ at a point $s$ in $S, \omega_{T}(s)=\omega(T, s)$, is the supremum over all positive $\alpha$ satisfying the following: for every neighborhood $U$ of $s$ there is a function $f$ of norm one in $C(S)$, which vanishes outside of $U$, with $\|T f\| \geqq \alpha$. The operator $T$ is almost diffuse if the set of diffusion points of $T, D(T)=\omega_{t}^{-1}(0)$, is dense and $T$ is countably almost diffuse if the set of concentration points, $\gamma(T)$ $=\omega_{t}^{-1}(0, \infty)$, is countable.

It is easy to show that the set $\omega_{T}^{-1}([a, \infty))$ is a closed set, so $\omega_{T}(s)$ is a nonnegative upper semicontinuous function of $s$ which is bounded by $\|T\|$. Consequently $D(T)=\omega_{T}^{-1}(0)$ is a $G_{\delta}$ and thus, if $D(T)$ is dense, i.e. if $T$ is almost diffuse, its complement $\gamma(T)$ is of first category [6, Theorem 1, p. 437]. In general any nonnegative upper semicontinuous function may occur as the oscillation of a linear operator. For example let $h$ be a nonnegative upper semicontinuous function on $[0,1]$. Define $A$ mapping $C[0,1]$ to $B[0,1]$, the bounded functions on $[0,1]$ with

Received by the editors March 5, 1971.

AMS 1970 subject classifications. Primary 47A55, 47B30, 47B99, 40H05.

Key words and phrases. Bounded linear operator on $C(S)$, oscillation of an operator, Fredholm operator, ramming operator, weakly compact operator, strictly singular operator, $\varphi_{+}$operator, conservative summability method, coercive summability method.

Copyright (C) 1972, American Mathematical Society 
the supremum norm, by $A f(s)=h(s) f(s)$. Since the range of $A$ is separable there is a linear isometry $B$ of it into $C[0,1][1$, p. 185]. The operator $B A$ on $C[0,1]$ has $\omega(B A, \cdot)=h(\cdot)$. By [11, Corollary 3, p. 288], or [12, Theorem 1, p. 656], a similar example can be constructed on $C(S)$ for any uncountable compact metric space $S$.

A minor error, which has repeatedly arisen in connection with almost diffuse operators, concerns the validity of the equation

$$
\|I+T\|=1+\|T\|
$$

which holds for $I$ the identity operator and for all almost diffuse operators $T$ on $C(S)$ [6, Theorem 3, p. 445]. The relation (1) was first proved for compact operators $T$ on $C[0,1]$ by Daugavet; the review in the Mathematical Reviews indicated that (1) was probably true for compact $T$ on $C(S)$ [3]. Again, see a remark given on p. 446 of [6]. Actually, their Theorem 3 is correct and the map given as a counterexample is not almost diffuse. In connection with his study of another class of operators, Krasnosel'skii claimed that (1) holds for all compact $T$ on $C(S), S$ any compact metric space [9]. If $S$ is a compact Hausdorff space with an isolated point $s_{0}$, then the map $K f=-f\left(s_{0}\right) \chi_{s_{0}}$ on $C(S)$ is compact but $\|I+K\|=1$. The remarks above are correct when $S$ has no isolated points and need a simple modification otherwise.

We introduce a subclass of the countably almost diffuse operators distinguished by a basic property.

Definition 1. Let $S$ be a compact Hausdorff space, $X$ a Banach space and $T: C(S) \rightarrow X$ a bounded linear operator. Then $T$ is a $c_{0}$ operator if the nonzero values in $\{\omega(T, s): s$ in $S\}$ are finite or a sequence converging to zero.

The set of $c_{0}$ operators is a closed subspace. A $c_{0}$ operator when multiplied on the left by a bounded linear operator is still a $c_{0}$ operator. As we will see in Example 1 the $c_{0}$ operators on $C(S)$ do not form a two-sided ideal.

The following type of operator was studied by Krasnosel'skir [9]: for $S$ a compact metric space and $T$ a bounded linear operator on $C(S)$ (or from $C(S)$ into $X$ ), $T$ is ramming if $\left\|T f_{n}\right\| \rightarrow 0$ for each uniformly bounded sequence of functions $\left\{f_{n}\right\}$ in $C(S)$ with nonoverlapping supports converging to a point in $S$.

THEOREM 1. Let $T: C(S) \rightarrow X$ be a bounded linear operator.

(a) If $T$ is weakly compact, then $T$ is a $c_{0}$ operator.

(b) If $S$ is a compact metric space and $T$ is a ramming operator, then $T$ is a $c_{0}$ operator. In general a $c_{0}$ operator is not ramming, but if $\omega(T, s)=0$ for all nonisolated points $s$, then $T$ is ramming.

Proof. Suppose $T$ is weakly compact and assume it is not a $c_{0}$ operator. Then we can find a sequence $\left\{s_{n}\right\}$ of distinct points of $S$ and a pairwise disjoint sequence $\left\{O_{n}\right\}$ of open sets with $s_{n}$ in $O_{n}$ and $\omega\left(T, s_{n}\right)>\varepsilon>0$ for all $n$. Since $\omega\left(T, s_{n}\right)>\varepsilon$, there is a function $f_{n}$ in $C(S)$ of norm one which vanishes outside of $O_{n}$ and which satisfies $\left\|T f_{n}\right\|>\varepsilon$. Consider the subspace $M=\overline{\operatorname{sp}}\left(f_{n}\right)$. The map $A: c_{0} \rightarrow M$ by 
$A\left(\left\{\alpha_{n}\right\}\right)=\sum \alpha_{n} f_{n}$ is easily shown to be a linear isometry of $c_{0}$ onto $M$. The restriction $\left.T\right|_{M}$ of $T$ to $M$ is weakly compact, $M$ is isomorphic to $c_{0}$, and thus $\left.T\right|_{M}$ is compact $[10,10$ Corollary $]$; the sequence $\left\{f_{n}\right\}$ converges weakly to zero, hence $\left\{T f_{n}\right\}$ converges to zero in norm. This contradiction shows that $T$ is a $c_{0}$ operator.

Suppose that $T: C(S) \rightarrow X, S$ a compact metric space, is ramming and assume that it is not a $c_{0}$ operator. There is a sequence $\left\{s_{n}\right\}$ of distinct points of $S$ with $\omega\left(T, s_{n}\right)>\varepsilon>0$, for all $n$, and by passing to a subsequence we may suppose that $\left\{s_{n}\right\}$ converges to a point $s_{0}$. By passing to a further subsequence we can find pairwise disjoint neighborhoods $S\left(s_{n}, r_{n}\right)$ with $r_{n} \rightarrow 0$. By assumption there is a function $f_{n}$ of norm one in $C(S)$ with support in $S\left(s_{n}, r_{n}\right)$ for which we have $\left\|T f_{n}\right\|>\varepsilon$. Because $T$ is ramming we must have $\left\|T f_{n}\right\| \rightarrow 0$, a contradiction.

Suppose that $T$ has $\omega(T, s)=0$ for all nonisolated points $s$. Let $\left\{f_{n}\right\}$ be a sequence of uniformly bounded functions in $C(S)$ with pairwise disjoint supports $\left\{U_{n}\right\}$ converging to $s_{0}$. Since $s_{0}$ is not isolated, given $\varepsilon>0$ there is a neighborhood $U$ of $s_{0}$ with $\|T f\| \leqq \varepsilon\|f\|$ for all $f$ in $C(S)$ with support in $U$. The sequence $\left\{U_{n}\right\}$ is eventually in $U$ which tells us that $\left\|T f_{n}\right\|$ is eventually bounded by $\varepsilon\left\|f_{n}\right\|$, i.e. $\left\|T f_{n}\right\| \rightarrow 0$ and $T$ is ramming. The example given below will show that a $c_{0}$ operator is not necessarily ramming. Note that the operator $T$ defined on $C[0,1]$ by $T f=f(0) 1$ is ramming and has $\omega(T, 0)=1$.

EXAMPLE 1. Consider the operator $C$ corresponding to $(C, 1)$ summability on $c$, i.e. $C\left(\left\{a_{n}\right\}\right)=\left\{\left(a_{1}+\cdots+a_{n}\right) / n\right\}$. Writing $c=C(S), S=\{\infty, 1,2,3, \ldots\}$ the onepoint compactification of the positive integers, $C$ is a $c_{0}$ operator on this $C(S)$ because $\omega(C, n)=\left\|C e_{n}\right\|=1 / n, e_{n}=\left\{\delta_{j n}\right\}_{j=1}^{\infty}$. Define $B$ on $C$ by

$$
B\left(\left\{a_{n}\right\}\right)=\left(a_{1}, a_{2}, a_{2}, a_{3}, a_{3}, a_{3}, a_{4}, a_{4}, a_{4}, a_{4}, a_{4}, a_{4}, a_{5}, \ldots\right) .
$$

Even though $B$ is an isometry on $c, C B$ is not a $c_{0}$ operator since $\left\|C B e_{n}\right\|=\frac{1}{2}$. Consider the sequence of functions in $c, f_{n}=\left\{f_{n}(j)\right\}$,

$$
\begin{aligned}
f_{n}(j) & =0, & & 1 \leqq j<2^{n-2} \\
& =1, & & 2^{n-2} \leqq j \leqq 2^{n-1}, \\
& =0, & & 2^{n-1}<j .
\end{aligned}
$$

The sequence $\left\{f_{n}\right\}$ is a uniformly bounded sequence of functions with pairwise disjoint supports converging to $\infty$, but as we have $\left\|C f_{n}\right\| \geqq \frac{1}{2}$ the operator $C$ is not ramming. This idea can easily be extended to operators on, say, $C[0,1]$. Define $T$ on $C[0,1]$ by

$$
\begin{aligned}
T f(t) & =\frac{1}{n} \sum_{j=1}^{n} f(1 / j), & & t=1 / n, \\
& =f(0), & & t=0, \\
& =\text { linear } & & \text { otherwise. }
\end{aligned}
$$

It is easy to show that $T$ is a $c_{0}$ operator on $C[0,1]$ but is not ramming. 
A characterization of almost diffuse operators given in [6, Proposition 1, p. 436] can be restated in the form: A bounded linear operator $T$ on $C[0,1]$ is almost diffuse if and only if given a closed ideal $I$ in $C[0,1]$ and $\varepsilon>0$ there is a closed ideal $J \subseteq I$ with $\left\|\left.T\right|_{J}\right\| \leqq \varepsilon$. There is a provocative similarity between this and a characterization of strictly singular operators, keeping in mind that all closed ideals in $C[0,1]$ are infinite dimensional, namely [7, III.2.1 Theorem, p. 84]: For a bounded linear operator $T: X \rightarrow Y$, between Banach spaces, the following are equivalent: (1) $T$ is strictly singular. (2) For each infinite dimensional closed subspace $M$ of $X$ and $\varepsilon>0$ there is a closed infinite dimensional subspace $N \subseteq M$ with $\left\|\left.T\right|_{N}\right\| \leqq \varepsilon$. (3) For each infinite dimensional closed subspace $M$ of $X$ there is a closed infinite dimensional subspace $N \subseteq M$ with $\left.T\right|_{N}$ compact. The operator of Example 1(a) of [6] is almost diffuse, in fact a $c_{0}$ operator, but is not compact when restricted to any closed ideal in $C[0,1]$, showing that we cannot expect a condition similar to (3), with closed subspaces replaced by closed ideals, to characterize almost diffuse operators. Another possibility is suggested by a class of operators studied by Herman [8]; those operators described by condition (3) above with "compact operator" replaced by "weakly compact operator." Our example below shows that $T$ may be a $c_{0}$ operator on $C[0,1]$ and yet have $\left.T\right|_{I}$ not weakly compact for any ideal $I$ in $C[0,1]$.

EXAmple 2. For $a$ in $(0,1)$ define the operator $T_{a}$ on $C[0,1]$ by

$$
\begin{aligned}
T_{a} f(t) & =f(a), & & 0 \leqq t \leqq a, \\
& =\frac{1}{t-a} \int_{a}^{t} f(x) d x, & & a<t \leqq 1 .
\end{aligned}
$$

The operator obtained by setting $a=0$ is discussed in [6, Example 2, p. 443]. The oscillation $\omega\left(T_{a}, s\right)$ is zero for $s \neq a$, so $T_{a}$ is a $c_{0}$ operator. Let $c$ and $d$ be given satisfying $0<c<a<d<1$ and define the functions $f(a, n, \cdot)$, for $n$ with $a+1 / n$ $<(a+d) / 2$, by

$$
\begin{aligned}
f(a, n, t) & =0, & & 0 \leqq t \leqq a, \\
& =1, & & t=a+1 / n, \\
& =1, & & t=(a+d) / 2, \\
& =0, & & d \leqq t \leqq 1, \\
& =\text { linear } & & \text { otherwise. }
\end{aligned}
$$

We compute

$$
\begin{aligned}
T_{a} f(a, n, t) & =0, & & 0 \leqq t \leqq a, \\
& =1-1 / 2 n(t-a), & & a+1 / n \leqq t \leqq(a+d) / 2 .
\end{aligned}
$$

For $a<t \leqq(a+d) / 2$ we have $T_{a} f(a, n, t) \rightarrow 1$ as $n \rightarrow \infty$, but $T_{a} f(a, n, a)=0$. Thus $\left\{T_{a} f(a, n, \cdot)\right\}$ cannot have a weakly convergent subsequence and $T_{a}$ is not weakly compact. 
For our example we choose $\left\{a_{n}\right\}$ a sequence of distinct points dense in $(0,1)$ and define

$$
T=\sum \frac{1}{2^{n}} T_{a_{n}}
$$

a $c_{0}$ operator since it is the limit of sums of $c_{0}$ operators. Let $I$ be a given closed nonzero ideal in $C[0,1] ; I=\{f$ in $C[0,1]: f(F)=0\}, F$ a closed subset of $[0,1]$. For some index $j, a_{j}$ does not belong to $F$. Let $\delta=1 / 2^{j+2}$ and choose $N$ so that $\sum_{N+1}^{\infty} 1 / 2^{n}<\delta$. There is a subinterval $[c, d]$ of $[0,1]$ which is disjoint from $F$ and contains $a_{j}$ in its interior but does not contain $a_{n}$ for $n \neq j, 1 \leqq n \leqq N$. The functions $\mathrm{g}_{m}(\cdot)=f\left(a_{j}, m, \cdot\right)$, defined for $m$ satisfying $a_{j}+1 / m<\frac{1}{2}\left(a_{j}+d\right)$, belong to $I$. Let $J=\{f$ in $C[0,1]: f([0,1]-(c, d))=0\}$. For $n \neq j$ and $1 \leqq n \leqq N$ the operator $\left.T_{a_{n}}\right|_{J}$ is compact because the Volterra operator $g(t) \rightarrow \int_{a_{n}}^{t} g(x) d x$ is compact on $J$ and the operator $g(t) \rightarrow g(t) /\left(t-a_{n}\right)$ is a bounded linear operator on $J$. Thus the sequence $\left\{T_{a_{n}} \cdot g_{m}(\cdot)\right\}_{m=1}^{\infty}$ is an equicontinuous family of functions [5, Theorem 7, p. 266]. Hence for $t$ close enough to $a_{j}$,

$$
\left|T_{a_{n}} g_{m}(t)-T_{a_{n}} g_{m}\left(a_{j}\right)\right|<\delta /\left(\sum_{j \neq p \leqq N} 2^{p}\right)
$$

holds uniformly for all $g_{m}$ and for $n \neq j, 1 \leqq n \leqq N$. Then

$$
\begin{aligned}
&\left|\frac{1}{2^{j}} T_{a_{j}} g_{m}(t)-\frac{1}{2^{j}} T_{a_{j}} g_{m}\left(a_{j}\right)-\left(T g_{m}(t)-T g_{m}\left(a_{j}\right)\right)\right| \\
& \leqq 2 \sum_{N+1}^{\infty} 1 / 2^{n}+\sum_{j \neq n \leqq N} 1 / 2^{n}\left|T_{a_{n}} g_{m}(t)-T_{a_{n}} g_{m}\left(a_{j}\right)\right| \leqq 3 \delta ;
\end{aligned}
$$

thus

$$
\left|T g_{m}(t)-T g_{m}\left(a_{j}\right)\right| \geqq\left|\frac{1}{2^{j}} T_{a,} g_{m}(t)-\frac{1}{2^{j}} T_{a_{j}} g_{m}\left(a_{j}\right)\right|-3 \delta .
$$

For $a_{j}<t \leqq \frac{1}{2}\left(a_{j}+d\right)$ the right-hand side of (2) tends to $1 / 2^{j}-3 \delta=1 / 2^{j+2}$ as $m$ tends to infinity. We see that no subsequence of $\left\{\operatorname{Tg}_{m}(\cdot)\right\}$ can converge weakly to a function continuous on $[0,1]$ and conclude that $\left.T\right|_{I}$ is not weakly compact.

The family of those operators on $C(S)$ which have a finite dimensional null manifold and a closed range (i.e. those $T$ with index $\mathscr{K}(T)<\infty$, sometimes called $\varphi_{+}$operators) is stable under perturbation by weakly compact operators since, on $C(S)$, the weakly compact operators and strictly singular operators coincide [7], [13]. This will not be true if we enlarge our perturbing class to the class of $c_{0}$ operators because a $c_{0}$ operator may have uncountably many eigenvalues; see Example 2 of [6] and Theorem V.1.8 of [7]. We introduce a set of operators which contain the $\varphi_{+}$operators and which are stable under perturbation by $c_{0}$ operators.

DEFinition 2. Let $S$ be a compact Hausdorff space, $X$ a Banach space and $T: C(S) \rightarrow X$ a bounded linear operator. The operator $T$ is concentrated if $\omega(T, s)$ has only finitely many zeros and is otherwise bounded away from zero. 
TheOREM 2. A bounded linear operator $T: C(S) \rightarrow X$ has a finite dimensional null manifold and a closed range if and only if there are a finite number of points $s_{1}, \ldots, s_{n}$ in $S$ with the property that, for $J=\left\{f\right.$ in $\left.C(S): f\left(s_{1}\right)=\cdots=f\left(s_{n}\right)=0\right\},\left.T\right|_{J}$ has a bounded inverse. If $T$ has a finite dimensional null manifold and a closed range, then $T$ is concentrated.

Proof. Let $J$ be as above and suppose that $\left.T\right|_{J}$ has a bounded inverse. Then the range of $T$ is closed [7, V.1.5 Lemma, p. 112], and the dimension of the null manifold of $T$ cannot exceed $n$.

Conversely suppose that $T$ has a null manifold $N$ of dimension $n$ and a closed range. Choose any $f_{1}$ in $N$ with $1=\left\|f_{1}\right\|=f_{1}\left(s_{1}\right)$. Any $g$ in $N$ can be written as $h+g\left(s_{1}\right) f_{1}, h\left(s_{1}\right)=0$, and so we can choose $f_{2}$ in $N$ with $f_{2}\left(s_{1}\right)=0,1=\left\|f_{2}\right\|=f_{2}\left(s_{2}\right)$. Any $g$ in $N$ can be written as $h+g\left(s_{1}\right) f_{1}+\left[g\left(s_{2}\right)-g\left(s_{1}\right) f_{1}\left(s_{2}\right)\right] f_{2}, h\left(s_{1}\right)=h\left(s_{2}\right)=0$. Continue, obtaining $f_{1}, \ldots, f_{n}$ in $C(S), N=\operatorname{sp}\left(f_{1} \cdots f_{n}\right)$ and $s_{1}, \ldots, s_{n}$ in $S$, $f_{i}\left(s_{j}\right)=\delta_{i j}$ for $j \leqq i$. Let $J=\left\{f\right.$ in $\left.C(S): f\left(s_{1}\right)=\cdots=f\left(s_{n}\right)=0\right\}$. Since the map $f \rightarrow\left(f\left(s_{1}\right), \ldots, f\left(s_{n}\right)\right)$ is a one-to-one linear map of $N$ into the set of all $n$-tuples of scalars with the maximum norm, it has a bounded inverse and there is a constant $a>0$ with $a\|f\| \leqq \max \left|f\left(s_{i}\right)\right|$ for all $f$ in $N$. For $g$ in $J$ and $f$ in $N,\|g-f\|$ $\geqq \max \left|g\left(s_{i}\right)-f\left(s_{i}\right)\right| \geqq a\|f\| ;$ also, $\|g-f\| \geqq\|g\|-\|f\|$. Thus

$$
\|g-f\| \geqq \max (a\|f\|,\|g\|-\|f\|) \geqq(a / a+1)\|g\| .
$$

For $g$ in $J$, using IV.1.6 Theorem, p. 98 of [7],

$$
\|T g\| \geqq \gamma(T) d(g, N) \geqq(a \gamma(T) / a+1)\|g\| .
$$

It follows that $\left.T\right|_{J}$ has a bounded inverse. Under these circumstances we have seen that for $s \notin\left\{s_{1}, \ldots, s_{n}\right\}$ and $U$ any neighborhood of $s$ disjoint from $\left\{s_{1}, \ldots, s_{n}\right\}$, $\|T f\| \geqq(a \gamma(T) / a+1)\|f\|$ whenever the support of $f$ is contained in $U ; \omega(T, s)$ $\geqq a \gamma(T) / a+1$ and $T$ is concentrated.

EXAMPLE 3. A concentrated operator need not have a closed range or a finite dimensional null manifold. Define $A$ on $c$ by the infinite matrix

$$
\left[\begin{array}{rrrrrrrrrr}
1 & 1 & -1 & 0 & 0 & 0 & 0 & 0 & 0 & \cdots \\
1 & 0 & 0 & 1 & -1 & 0 & 0 & 0 & 0 & \cdots \\
1 & 0 & 0 & 0 & 0 & 1 & -1 & 0 & 0 & \cdots \\
\vdots & & & & & & & & &
\end{array}\right]
$$

We again regard $c$ as $C(S), S=\{\infty, 1,2,3, \ldots\}$ the one point compactification of the integers. It is easy to show that $A$ has closed range (it is onto $c$ ) and the null manifold of $A$ is infinite dimensional; $A$ is concentrated since $\left\|A e_{n}\right\|=1$ for the 
vector $e_{n}=\left\{\delta_{j n}\right\}$. Define $B$ on $c$ by the infinite matrix

$$
\left[\begin{array}{rrrrrrrrr}
-1 & 0 & 0 & 0 & 0 & 0 & 0 & 0 & \cdots \\
1 & -1 & 0 & 0 & 0 & 0 & 0 & 0 & \cdots \\
1 & 1 & -1 & 0 & 0 & 0 & 0 & 0 & \cdots \\
1 & 0 & 1 & -1 & 0 & 0 & 0 & 0 & \cdots \\
1 & 0 & 0 & 1 & -1 & 0 & 0 & 0 & \cdots \\
\vdots & & & & & & & &
\end{array}\right] .
$$

The operator $B$ is one-to-one and its range is not closed (the vectors $x^{n}=(1 / n$, $2 / n, \ldots,(n-1) / n, 1,(n-1) / n, \ldots, 2 / n, 1 / n, 0,0,0, \ldots)$ have $\left\|x^{n}\right\|=1$, but $\left\|B x^{n}\right\|$ $=2 / n) ; B$ is concentrated since $\left\|B e_{n}\right\|=1$. The product $B A$ is a concentrated operator with infinite dimensional null manifold and nonclosed range.

THEOREM 3. If $T: C(S) \rightarrow X$ is a concentrated operator and $K: C(S) \rightarrow X$ is a $c_{0}$ operator, then $T+K$ is a concentrated operator.

Proof. Suppose that $T+K$ is not concentrated and let $\varepsilon>0$ be given. There is a sequence $\left\{s_{n}\right\}$ of distinct points of $S$ with $\omega\left(T+K, s_{n}\right)<\varepsilon / 2$ and by passing to a subsequence we may also suppose that $\omega\left(K, s_{n}\right)<\varepsilon / 2$. There is a neighborhood $U_{n}$ of $s_{n}$ with $\|(T+K) f\| \leqq \varepsilon / 2\|f\|$ for $f$ with support in $U_{n}$ and there is a neighborhood $V_{n}$ of $s_{n}$ with $\|K f\| \leqq \varepsilon / 2\|f\|$ for $f$ with support in $V_{n}$. For $f$ in $C(S)$ with support in $V_{n} \cap U_{n}$,

$$
\|T f\| \leqq\|(T+K) f\|+\|K f\| \leqq \varepsilon\|f\| .
$$

Hence $\omega\left(T, s_{n}\right) \leqq \varepsilon$. That is, for each $\varepsilon>0$ there are infinitely many points $s_{n}$ with $\omega\left(T, s_{n}\right) \leqq \varepsilon$, a contradiction since $T$ is concentrated.

Recall that a summability method is an infinite matrix $A=\left(a_{i j}\right)$ of scalars. For a sequence $\left\{b_{j}\right\}$ of scalars, $A\left(\left\{b_{j}\right\}\right)=\left\{\sum_{j=1}^{\infty} a_{i j} b_{j}\right\}$ is defined whenever the sums $\sum_{j=1}^{\infty} a_{i j} b_{j}$ all converge. A matrix $A$ sums a sequence $\left\{b_{j}\right\}$ if $A\left(\left\{b_{j}\right\}\right)$ is convergent, i.e. belongs to $c$, and is conservative if it sums every sequence in $c$. By the SilvermanToeplitz conditions $[15$, p. 118$]$ a conservative matrix, when restricted to $c$, is a bounded linear operator with $\|A\|=\sup _{i} \sum_{j=1}^{\infty}\left|a_{i j}\right|$ finite. As we have done previously, we will identify $c$ with $C(S), S=\{\infty, 1,2, \ldots\}$ the one point compactification of the positive integers, and will set $e_{n}=\left\{\delta_{j n}\right\}$. A conservative summability matrix $A$ can be regarded as a bounded linear map on a space of type $C(S)$ and there has an oscillation $\omega(A, \cdot)$ which we will show is connected with its summability properties.

For $T: c \rightarrow X$ a bounded linear operator, $\omega(T, n)=\left\|T e_{n}\right\|$ and $\omega(T, \infty)=$ $\lim _{n \rightarrow \infty}\left\|\left.T\right|_{M_{n}}\right\|$ where $M_{n}=\{f$ in $c: f(j)=0$ for $1 \leqq j \leqq n\}$. The oscillation is most interesting at infinity.

Theorem 4 is unusual in that it characterizes those summability methods which are coercive, i.e. which sum every bounded sequence, among all the bounded linear operators on $c$ not just among the conservative summability methods. 
THEOREM 4. Suppose that $T: c \rightarrow c$ is a bounded linear operator. Then $\omega(T, \infty)=0$ if and only if $T$ is a summability method which sums every bounded sequence, i.e. if and only if $T$ is a conservative summability method which is a compact operator on $c$.

Proof. We begin by showing that a bounded linear operator $L: c \rightarrow c$ is compact if and only if $\lim \left\|\left.L\right|_{N_{n}}\right\|=0$, where $N_{n}=\{x$ in $c: x(j)=0,1 \leqq j \leqq n$, and $x(\infty)=0\}$. Suppose that $L$ is compact and assume that $\left\|\left.L\right|_{N_{n}}\right\|$ does not converge to zero. There is an $\varepsilon>0$ and a sequence $z_{n_{i}}$ with $\left\|z_{n_{i}}\right\|=1, z_{n_{i}}$ belongs to $N_{n_{i}}$ and $\left\|L z_{n_{i}}\right\| \geqq \varepsilon$. But the sequence $\left\{z_{n_{i}}\right\}$ converges weakly to zero and, since $L$ is compact, $\left\{L z_{n_{i}}\right\}$ must converge to zero in norm, which is a contradiction. Conversely suppose that $\lim \left\|\left.L\right|_{N_{n}}\right\|=0$. Define $P_{n}$ on $c$ by $P_{n}\left\{b_{j}\right\}=\left(\lim b_{j}\right)(1,1,1, \ldots)+\sum_{i=1}^{n}\left(b_{i}-\lim b_{j}\right) e_{i}$. Then $\left\|L-L P_{n}\right\| \leqq\left\|I-P_{n}\right\|\left\|\left.L\right|_{N_{n}}\right\| \leqq 2\left\|\left.L\right|_{N_{n}}\right\|$. The bounded linear operators $L P_{n}$ are compact because their ranges are finite dimensional and the limit $L$ of these compact operators is compact.

Let $T: c \rightarrow c$ be a bounded linear operator on $c$ with $\omega(T, \infty)=0$. The general form of an operator on $c$ is well known [14], to $T$ there corresponds a sequence of continuous linear functionals on $c, x_{n}^{*}=\left\{a_{n j}\right\}_{j=0}^{\infty}$ in $l$, converging in the $c$-topology on $l$, with $T x=\left\{x_{n}^{*}(x)\right\}$ for each $x$ in $c$. (Recall that $x_{n}^{*}\left(\left\{b_{j}\right\}\right)=a_{n 0} \lim b_{j}+\sum_{j=1}^{\infty} a_{n j} b_{j}$.) Define $y_{m}$ in $c$ by

$$
\begin{aligned}
y_{m}(j) & =0, & & 1 \leqq j \leqq m \\
& =1, & & m<j
\end{aligned}
$$

Since $\omega(T, \infty)=0,\left\|T y_{m}\right\| \rightarrow 0$ and thus $a_{n 0}=0$ for all $n$, which is to say that $T$ is a (conservative) summability method. Because $T$ is a summability method, $\left\|\left.T\right|_{N_{n}}\right\|$ $=\left\|\left.T\right|_{M_{n}}\right\|, M_{n}=\{x$ in $c: x(j)=0,1 \leqq j \leqq n\}$. Hence if $T$ has $\omega(T, \infty)=0$, then $T$ is a summability method with $0=\lim \left\|\left.T\right|_{M_{n}}\right\|=\lim \left\|\left.T\right|_{N_{n}}\right\|$ and $T$ is therefore compact. Conversely if $T$ is a compact summability method then $0=\lim \left\|\left.T\right|_{N_{n}}\right\|=\lim \left\|\left.T\right|_{M_{n}}\right\|$ $=\omega(T, \infty)$.

To complete the proof we need to show that a summability method is coercive, i.e. sums every bounded sequence, if and only if it is compact. This is probably well known and is given in [15, p. 243, Problem 43] for a special type of matrix. We indicate a proof. Suppose that $T: c \rightarrow c$ is a compact summability method. Then $T$ has the form described above, $T x=\left\{x_{n}^{*}(x)\right\}$, and the sequence $\left\{x_{n}^{*}\right\}$ converges in norm to the functional $x^{*}$ [14]. Then for any bounded sequence $\left\{b_{j}\right\}, \lim x_{n}^{*}\left(\left\{b_{j}\right\}\right)$ $=x^{*}\left(\left\{b_{j}\right\}\right)$ and $T$ is coercive. On the other hand suppose that $T$ is a coercive summability matrix. The Silverman-Toeplitz conditions show that $T: m \rightarrow c$ is a bounded linear operator (with $\|T\|=\left\|\left.T\right|_{c}\right\|=\sup \left\|x_{n}^{*}\right\|$ ). Let $\left\{y_{n}^{*}\right\}$ be a bounded sequence in $c^{*}$; it contains a subsequence $\left\{y_{n_{i}}^{*}\right\}$ converging to $y^{*}$ in the weak topology [5, Theorem 1, p. 426]. The sequence $\left\{T^{*} y_{n_{i}}^{*}\right\}$ is then a weak* convergent sequence in $m^{*}$ and so it converges weakly [4, p. 109]. Hence $T^{*}$ is a weakly compact operator and therefore $T$ is also weakly compact. But then as a map on $c, T$ is weakly compact and $\left.T\right|_{c}$ is therefore compact [10, 10 Corollary]. 
Albert Wilansky has pointed out that the matrix $A$ of Example 3 occurs in his paper Topological divisors of zero and Tauberian theorems, Trans. Amer. Math. Soc. 113 (1964), 240-251, and that there it is also mentioned that coercive and compact are equivalent (see Theorem 4), with a reference to A. Wilansky and K. Zeller, Banach algebra and summability, Illinois J. Math. 2 (1958), 378-385. Wilansky credits this result to E. K. Dorff in his unpublished Ph.D. thesis (Lehigh University, 1958).

\section{REFERENCES}

1. S. Banach, Théorie des opérations linéaires, Chelsea, New York, 1955. MR 17, 175.

2. E. Cheney and K. Price, Minimal projections, Approximation Theory, Academic Press, New York, 1970, pp. 261-289. MR 42 \#751.

3. I. Daugavet, $A$ property of completely continuous operators in the space $C$, Uspehi Mat. Nauk 18 (1963), no. 5 (113), 157-158. (Russian) MR 28 \#461.

4. M. Day, Normed linear spaces, Ergebnisse der Mathematik und ihrer Grenzgebiete, N. F., Heft 21, Academic Press, New York; Springer-Verlag, Berlin, 1962. MR 26 \#2847.

5. N. Dunford and J. T. Schwartz, Linear operators. I: General theory, Pure and Appl. Math., vol. 7, Interscience, New York and London, 1958. MR 22 \#8302.

6. C. Foias and I. Singer, Points of diffusion of linear operators and almost diffuse operators in spaces of continuous functions, Math. Z. 87 (1965), 434-450. MR 31 \#5093.

7. S. Goldberg, Unbounded linear operators: Theory and application, McGraw-Hill, New York, 1966. MR 34 \#580.

8. R. Herman, Generalizations of weakly compact operators, Trans. Amer. Math. Soc. 132 (1968), 377-386. MR 36 \#6976.

9. M. Krasnosel'skiī, A class of operators in a space of abstract continuous functions, Mat. Zametki 2 (1967), 599-604. MR 36 \#5733.

10. E. Lacey and R. Whitley, Conditions under which all the bounded linear maps are compact, Math. Ann. 158 (1965), 1-5. MR 30 \#3373.

11. A. Pełczyński, On simultaneous extension of continuous functions. A generalization of theorems of Rudin-Carleson and Bishop, Studia Math. 24 (1964), 285-304. MR 30 \#5184a.

12. - Some linear topological properties of separable function algebras, Proc. Amer. Math. Soc. 18 (1967), 652-660. MR 35 \#4737.

13. - On strictly singular and strictly cosingular operators. I: Strictly singular and strictly cosingular operators in $C(S)$-spaces, Bull. Acad. Polon. Sci. Sér. Sci. Math. Astronom. Phys. 13 (1965), 31-36. MR 31 \#1563.

14. E. Thorp and R. Whitley, Operator representation theorems, Illinois J. Math. 9 (1965), 595-601. MR 31 \#6126.

15. A. Wilansky, Functional analysis, Blaisdell, New York, 1964. MR 30 \#425.

Department of Mathematics, University of California, Irvine, California 92664 